\title{
Utilization of Er:YAG Laser in Retrieving and Reusing of Lithium Disilicate and Zirconia Monolithic Crowns in Natural Teeth: An In Vitro Study
}

\author{
Kinga Grzech-Leśniak ${ }^{1,2} \oplus$, Sompop Bencharit ${ }^{3,4,5, *} \mathbb{}$, Lenart Skrjanc ${ }^{6}$, Domen Kanduti ${ }^{7}$, \\ Jacek Matys ${ }^{2}$ and Janina Golob Deeb ${ }^{1}$ \\ 1 Department of Periodontology, School of Dentistry, Virginia Commonwealth University, Richmond, \\ VA 23298, USA; kgl@periocare.pl (K.G.-L.); jgolobdeeb@vcu.edu (J.G.D.) \\ 2 Department of Oral Surgery, Wroclaw Medical University, 50-367 Wrocław, Poland; jacek.matys@wp.pl \\ 3 Department of General Practice, School of Dentistry, Virginia Commonwealth University, Richmond, \\ VA 23298, USA \\ 4 Department of Oral and Maxillofacial Surgery, School of Dentistry, Virginia Commonwealth University, \\ Richmond, VA 23298, USA \\ 5 Department of Biomedical Engineering, College of Engineering, Virginia Commonwealth University, \\ Richmond, VA 23298, USA \\ 6 Faculty of Medicine, University of Ljubljana, 1000 Ljubljana, Slovenia; lenart.skrjanc@gmail.com \\ 7 Department of Periodontology. Faculty of Medicine, University of Ljubljana, 1000 Ljubljana, Slovenia; \\ domen.kanduti@mf.uni-lj.si \\ * Correspondence: sbencharit@vcu.edu
}

Received: 8 June 2020; Accepted: 22 June 2020; Published: 25 June 2020

Featured Application: This work demonstrates that an erbium-doped yttrium aluminum garnet laser (Er:YAG). laser can be used as a non-invasive crown retrieval tool for common all-ceramic crowns. The type of all-ceramic material has a significant impact on crown removal time. However, the cement thickness has less influence on retrieval time or temperature. The temperature increase during laser irradiation does not appear to be harmful or clinically significant.

Abstract: The study examined the effect of noninvasive crown retrieval/reuse process using an erbium-doped yttrium aluminum garnet laser (Er:YAG). Twenty-six extracted human teeth were prepared for a crown. The crown was milled using lithium disilicate (LD) and zirconia (Z) materials, $n=13$ per group, with three for scanning electron microscopy (SEM). The crown was luted using composite resin cement and subjected to a laser retrieval process. After the retrieval process, the crown was cleaned, re-cemented and laser-retrieved two more times, without and with additional tooth reduction mimicking clinical refreshment of dentin. Retrieval time and temperature were analyzed using analysis of variance (ANOVA). Surface changes were observed through SEM. The retrieval times were $267.1 \pm 130.43,220 \pm 79.09,277.1 \pm 126.44,368.4 \pm 136.14,355 \pm 159.39$, and $419.2 \pm 121.36 \mathrm{~s}$ for first, second, third LD and Z groups, respectively $(p=0.009)$. The maximal temperatures were 23.95.1 $\pm 1.89^{\circ} \mathrm{C}, 24.86 \pm 2.01{ }^{\circ} \mathrm{C}, 24.17 \pm 1.53^{\circ} \mathrm{C}, 22.88 \pm 1.51^{\circ} \mathrm{C}, 24.03 \pm 1.74{ }^{\circ} \mathrm{C}$, and $21.99 \pm 1.32{ }^{\circ} \mathrm{C}$ for first, second, third LD and Z groups, respectively $(p=0.006)$. Er:YAG laser crown removal is an effective retrieval tool for all-ceramic crowns. Minimal changes to teeth and crowns were observed following laser irradiation.

Keywords: Crown removal; Debond; Er:YAG; Laser; Lithium disilicate; Zirconia 


\section{Introduction}

Recent advancement of digital technologies, including intraoral scanning, computer-aided design and computer-aided manufacturing (CAD/CAM), as well as biomaterials, allows dentists to fabricate fixed prostheses such as a crown in a rapid streamline process [1-4]. These crowns, most frequently made of ceramic materials such as lithium disilicate or zirconia, are esthetically pleasing, with superior biocompatibility and physical properties as well as survival rates [5-7]. The crown material, especially lithium disilicate, can be bonded to the natural tooth abutment using acid etching and composite resin cement [8-10], while zirconia materials used to make these crowns are perhaps the strongest ceramic materials available in contemporary dentistry $[11,12]$. These physical strengths and bonding capabilities allow for long lasting restorations in the oral cavity. These clinically desirable properties however become a major obstacle when the crown needs to be removed $[13,14]$. When the crown retrieval is indicated due to recurrent dental caries, misfits, esthetic reasons, material damage or a need for endodontic therapy, a rotary handpiece with a cutting bur is a common conventional method to remove the crown. This process leads to reversible damage of the crown and possibly the tooth structure, resulting in a need for a new restoration, which can be a time consuming and costly process for both dentist and patient. Ideally, the dentist should be able to retrieve the crown from the abutment tooth without trauma and quickly without damaging the crown or tooth structure. The crown would then be re-cemented back onto the tooth. Such a process would reduce chair time, discomfort, time, frustration, and cost to both the provider and the patient.

The use of lasers for removal of restorative materials from natural teeth or dental implants is not new. High power lasers such as erbium-doped yttrium aluminum garnet laser (Er:YAG) have been used to retrieve all-ceramic restorations [15,16]. The energy wavelength of $2940 \mathrm{~nm}$ is passed through the translucent ceramic material with little absorption and targets the water and monomer molecules in the cement layer. The energized water and monomer molecules create an ablation cascade of cement and therefore the all-ceramic restorations can be retrieved without any structural damage to the crown or to the natural abutment tooth. Er:YAG laser has been used to remove composite resin restorations [17], veneers [18,19], orthodontic brackets [20,21] and crowns [22]. Recent studies used Er:YAG laser to retrieve lithium disilicate crowns from zirconia or titanium implant abutments [23-25] and zirconia crowns from zirconia implant abutments [25] in a few minutes without any damage to the crown or abutment materials. These studies also demonstrate the ability of the Er:YAG laser to retrieve and reuse all-ceramic (lithium disilicate or zirconia) crowns multiple times without any damages to the dental implant crown or abutment materials [23-25]. When applying Er:YAG laser at 2940 nanometer $(\mathrm{nm})$ wavelength to remove lithium disilicate crowns from dental implant abutments, the removal time ranged from $\sim 3$ to $5 \mathrm{~min}(\mathrm{~min})[23,24]$, and the temperature of the crowns appeared to increase by $4-6{ }^{\circ} \mathrm{C}[23,24]$. When similar set up and laser was used to remove zirconia crowns from implant abutments, the average removal time increased to almost $6 \mathrm{~min}$, while the temperature increased by $3-7^{\circ} \mathrm{C}$ [25]. The Er:YAG laser at $2940 \mathrm{~nm}$ wavelength does not seem to have any adverse effects toward lithium disilicate or zirconia crown or implant abutment materials [23-25]. However, repeated Er:YAG laser assisted crown retrieval seems to increase the amount of remaining cement in the implant crown $[24,25]$ and increase the surface roughness through roughening of feldspathic glaze porcelain on the zirconia implant crown surface [25]. While there is clinical and research evidence for removing all-ceramic crowns with a Er:YAG laser, there is little information available on the repeated laser-assisted crown retrievals and reuses for natural abutment teeth and of the effect different restorative materials may have on the length of laser irradiation needed for this process.

This study aimed to examine the effects of Er:YAG laser in the repeated crown retrieval process from natural teeth using the most common all-ceramic materials for crowns, namely lithium disilicate and zirconia. The hypothesis was that with an Er:YAG laser, retrieval of a lithium disilicate crown would take less time and result in higher intra-pulpal temperature compared to a zirconia crown. Additional goal was to examine any structural surface damage to the materials or teeth as a result of repeated irradiation during the crown retrieval process. 


\section{Materials and Methods}

\subsection{Specimen Preparation}

Specimen preparation process is shown in Figure 1. Twenty-six recently extracted human molars were used. Teeth were kept in distilled water during the waiting period for specimen preparation. Each tooth specimen was prepared past dentino-enamel junction into dentin to accommodate a lithium disilicate or zirconia crown with material thickness of approximately $1 \mathrm{~mm}(\mathrm{~mm})$ axially and $1.5 \mathrm{~mm}$ occlusally using a dental rotary handpiece and diamond burs with generous air and water coolant. A $0.5 \mathrm{~mm}$ deep chamfer finishing line was used. After the tooth preparation, the specimen was scanned using an intraoral scanner (Emerald, Planmeca, Helsinki, Finland). The crown was virtually designed using CAD software (Romexis version 5.1, Planmeca, Helsinki, Finland). The material thickness of $\sim 0.5 \mathrm{~mm}$ at the crown margin and $\sim 1-1.5 \mathrm{~mm}$ axially and occlusally was used. The cement gap was designed as $100 \mu \mathrm{m}$.

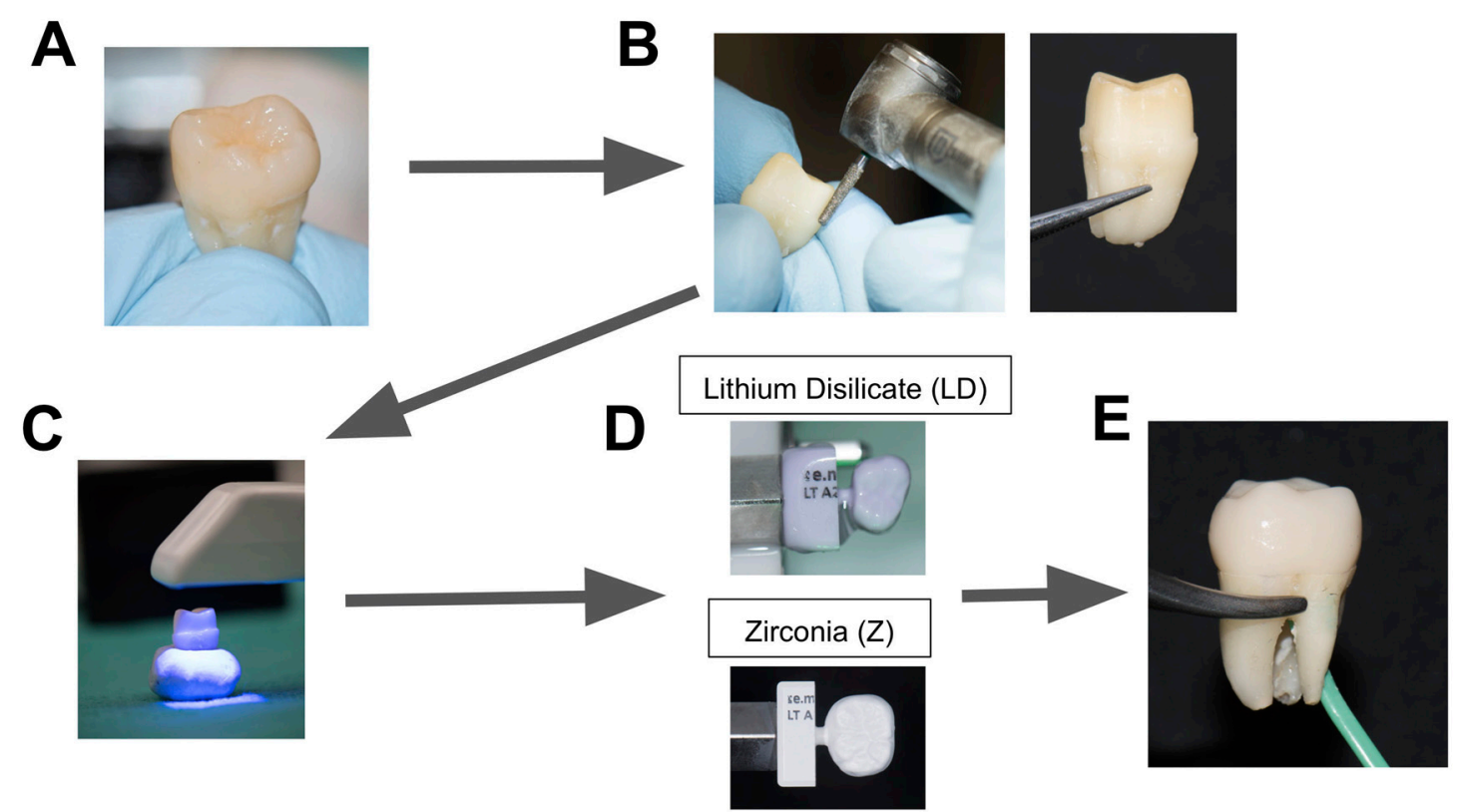

Figure 1. Specimen preparation. (A) Recently discarded extracted natural teeth were selected and cleaned, (B) the tooth was prepared for the crown, (C) the tooth was scanned using an intraoral scanner and the digital design was done, (D) the crown was milled, (E) the crown was cemented and the temperature probe was inserted.

For the lithium disilicate crown, the crown design was exported into a standard tessellation language (STL) format to be milled. A pre-sintered monolithic lithium disilicate block (IPS e.max CAD C14 LT A2, Ivoclar Vivadent, Schaan, Liechtenstein) was milled using a single spindle desktop milling machine (PlanMill 30S, Planmeca, Helsinki, Finland). The pre-sintered milled crown was then stained, characterized with feldspathic porcelain, and sintered using the manufacturer's recommended combined staining, glazing, and crystallization protocol in a ceramic furnace (Programat CS3, Ivoclar Vivadent, Schaan, Liechtenstein) for $\sim 15 \mathrm{~min}$ in the furnace or 45 min total time including the preheating and subsequent cooling of the furnace. The excess glaze around the margin was removed and the crown was polished. The luting protocol was based on the manufacturer's recommendation. The crown was tried to determine appropriate fitting and then luted with composite resin cement (Multilink Automix, Ivoclar Vivadent, Schaan, Liechtenstein). Briefly, the crown was steam-cleaned, etched with 5\% hydrofluoric acid (IPS Ceramic Etching Gel, Ivoclar Vivadent, Schaan, Liechtenstein) for $20 \mathrm{~s}$ (sec), rinsed with water and air dried, conditioned with silane for $60 \mathrm{~s}$ (Monobond Plus, Ivoclar Vivadent, Schaan, Liechtenstein) and then air dried. The abutment was primed for $30 \mathrm{~s}$ with a 
mixture of two primers per the manufacturer's recommendation (Primer A and B, Multilink, Ivoclar Vivadent, Schaan, Liechtenstein). The crown was pressed with finger-pressure for about $5 \mathrm{~min}$. Light polymerization for $20 \mathrm{~s}$ for each axial and occlusal surface was performed. The cemented specimen was kept in a 100\% humidity chamber using distilled water for at least $48 \mathrm{~h}$ before crown retrieval.

For the zirconia crown, the crown design was exported and milled using a single spindle desktop milling machine (PlanMill 30S, Planmeca, Helsinki, Finland) out of a monolithic $3 \mathrm{~mol} \%$ yttria stabilized tetragonal zirconia polycrystal (3Y-TZP) block (IPS e.max ZirCAD LT C17 A2, Ivoclar Vivadent, Schaan, Liechtenstein). The milled crown was sintered for $45 \mathrm{~min}$ per the manufacturer's recommendation using a sintering furnace (Programat CS4, Ivoclar Vivadent, Schaan, Liechtenstein). After sintering, the crown was characterized and stained using the same furnace (Programat CS4, Ivoclar Vivadent, Schaan, Liechtenstein). The intaglio surface of the glazed crowns was then again air-abraded with $50 \mu \mathrm{m}$ aluminum oxide particles. Excess glaze around the margin was removed. The crown was polished and tried on the abutment to determine the fitting. The crown was conditioned with silane containing primer for $60 \mathrm{~s}$ (Monobond Plus, Ivoclar Vivadent, Schaan, Liechtenstein) and then air dried. The composite resin cement (Multilink Automix, Ivoclar Vivadent, Schaan, Liechtenstein) and cementation process used were the same as the one for lithium disilicate crowns. The cemented specimen was kept in a 100\% humidity chamber using distilled water for at least $48 \mathrm{~h}$ before crown retrieval.

\subsection{Specimen Assignment, Treatment, and Retrieval Process}

The study workflow and grouping are shown in Figure 1. A total of 13 cemented crown specimens for each group were made. Grouping was assigned per material LD for lithium disilicate and $\mathrm{Z}$ for zirconia. Initially all 26 specimens underwent the laser-assisted crown retrieval process. One specimen from each group was removed for scanning electron microscopy (SEM) examination. These first retrieval attempts were recorded as LD1 and Z1.

After the first crown retrieval, the crowns and the abutment teeth were air abraded and rinsed with water. The crowns were then re-cemented using the same luting processes as described above. The second retrieval was performed under the same laser parameters and recorded as LD2 and Z2 attempt. The second retrieval attempt mimicked the clinical re-cementation process when no major alteration and adjustment of the natural tooth abutment is needed. One specimen from each group was again removed for SEM examination. Following the second crown retrieval, the crowns were again air abraded and rinsed with water. The abutment teeth were additionally reduced using a handpiece and diamond burs to simulate adjustments sometimes indicated following crown retrieval in clinical practice. Attempts were made to uniformly adjust the axial and occlusal surfaces of the teeth without disturbing the integrity of the margins.

Following the additional tooth reduction, teeth were scanned again using the same intraoral scanner to capture the surface area changes. The abutment surfaces and the new cement spaces were calculated using MeshMixer software (version 3.5, Autodesk, San Rafael, CA, USA).

The crowns were then re-cemented using the same cementation protocol per type of crown material, kept in a 100\% humidity chamber using distilled water for $48 \mathrm{~h}$ and retrieved. These retrieval attempts were recorded as LD3 and Z3. One specimen from each group was kept for SEM analysis.

\subsection{Laser Assisted Crown Retrieval Process}

An apical channel was prepared through the furcation of the tooth into the pulpal chamber to insert the thermometer probe for a microthermal couple element (Adv. Thermocouple Therm. w/RS232 Output Datalogger Type K- 800008, Super Scientific Works Pvt. Ltd., Gujarat, India) to measure the temperature changes during the laser-assisted crown retrieval process (Figures 1 and 2). The cemented crown/abutment specimen was mounted into a 3-D printed typodont with adjacent natural teeth to simulate a clinical situation during laser irradiation (Figure 2).

Similar to previous studies [23-25], Er:YAG laser (Lightwalker, Fotona, Slovenia) was set at: $300 \mathrm{~mJ}, 15 \mathrm{~Hz}, 4.5 \mathrm{~W}$, spot size $0.9 \mathrm{~mm}$, fluence per pulse $47.16 \mathrm{~J} / \mathrm{cm}^{2}$, power density $707.4 \mathrm{~W} / \mathrm{cm}^{2}$, 
operation mode: quantum square pulse (QSP) mode (50 $\mu$ s pulse duration) 2 water/2 air. Using a non-contact continuous motion tipless handpiece $\left(\mathrm{HO}_{2}\right.$, Lightwalker, Fotona, Slovenia), the crown was irradiated buccally, lingually, occlusally, and indirectly interproximally at a distance of 6-10 mm. Following 2 min of irradiation time, the crown was gently tapped with a traditional crown removal instrument on the buccal and lingual margins, simulating clinical access to those surfaces. If removal could not be achieved, the tooth was subjected to additional $30 \mathrm{~s}$ intervals of irradiation followed by the attempt of crown removal after each of the intervals. The irradiation time was recorded until the crown was successfully retrieved either by tapping, light digital manipulation or passively lifting off during the irradiation (Figure 2). Before initiating the crown removal, pulpal temperatures were recorded at the baseline and throughout the entire procedure at $30 \mathrm{~s}$ intervals until the crown was retrieved.
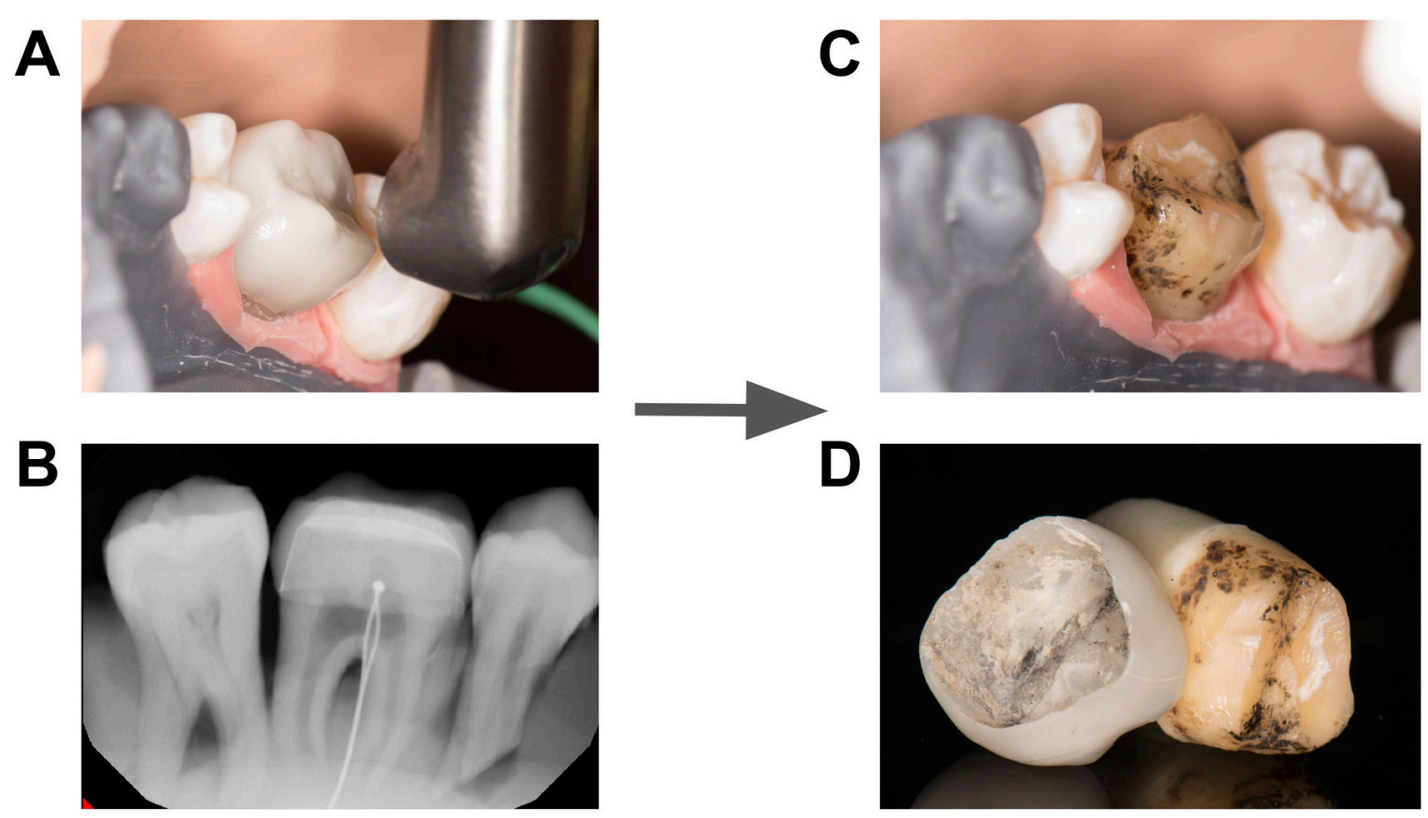

Figure 2. Laser-assisted crow removal. (A) Laser irradiation, (B) Radiograph showing temperature probe positioning, (C) Abutment after crown removal, and (D) crown and abutment specimens after crown removal.

After the first, second, and third crown retrieval experiment, one specimen for each group was withheld for SEM analysis. To examine the surface integrity of the crown after each irradiation and crown retrieval, the underlying intaglio surface of the crown was examined using scanning electron microscopy (SEM) analysis (JEOL 6610LV, JEOL, Japan). Similar to the previous studies [23-25], secondary electron imaging (SEI) to topography of the structure analysis was applied to enhance structural assessment and visualization of possible cracks using back-scattered electrons (BSE).

\subsection{Measurements and Statistical Analyses}

The lithium disilicate crown retrieval measurements included the first retrieval (LD1), the second retrieval (LD2: re-cemented without abutment adjustment), and the third retrieval (LD3: re-cemented with abutment adjustment). Similarly, the zirconia crown retrieval measurements included the first retrieval (Z1), the second retrieval (Z2: re-cemented without abutment adjustment), and the third retrieval (Z3: re-cemented with abutment adjustment). Data analysis was performed only on the specimens that had gone through all three retrievals $(n=10$ for each group). The crown retrieval time and the highest temperature from each group were statistically analyzed using analysis of variance (ANOVA, single factor) with level of significance, $\alpha=0.05$. The null hypotheses were that there was no statistical difference among the group of different materials, lithium disilicate and zirconia, and with different retrieval attempts $(1,2$, and 3). ANOVA with $\alpha=0.05$ was used to examine the statistical 
difference within the same material. $T$-test assuming unequal variances with $\alpha=0.05$ was used to determine the difference between materials for each retrieval. To examine the effects of tooth alteration in the third retrieval, Pearson correlations were calculated for the abutment surface and retrieval time for the first and third retrieval, as well as the cement layer and retrieval time/temperature.

\section{Results}

The summaries of the results are shown in Tables 1 and 2. The average crown retrieval times for lithium disilicate were $267.1 \pm 130.43,220 \pm 79.09,277.1 \pm 126.44 \mathrm{~s}$ for LD1, LD2, and LD3, respectively. On the other hand, the ones for zirconia were $368.4 \pm 136.14,355 \pm 159.39$, and $419.2 \pm 121.36 \mathrm{~s}$ for Z1, Z2, and Z3, respectively. There was a statistical difference overall (ANOVA, $p=0.009$ ); however, there was no statistical difference within the same material (ANOVA, $p=0.5$ for lithium disilicate, and $p=0.56$ for zirconia). When comparing the restorative materials, zirconia crown retrieval takes approximately 100-200 s longer than lithium disilicate one with statistical significance except for the first retrieval experiment ( $t$-test, $p=0.11, p=0.03$ and $p=0.02$ for first, second, and third retrieval, respectively).

Table 1. Er:YAG laser-assisted crown retrieval time (sec).

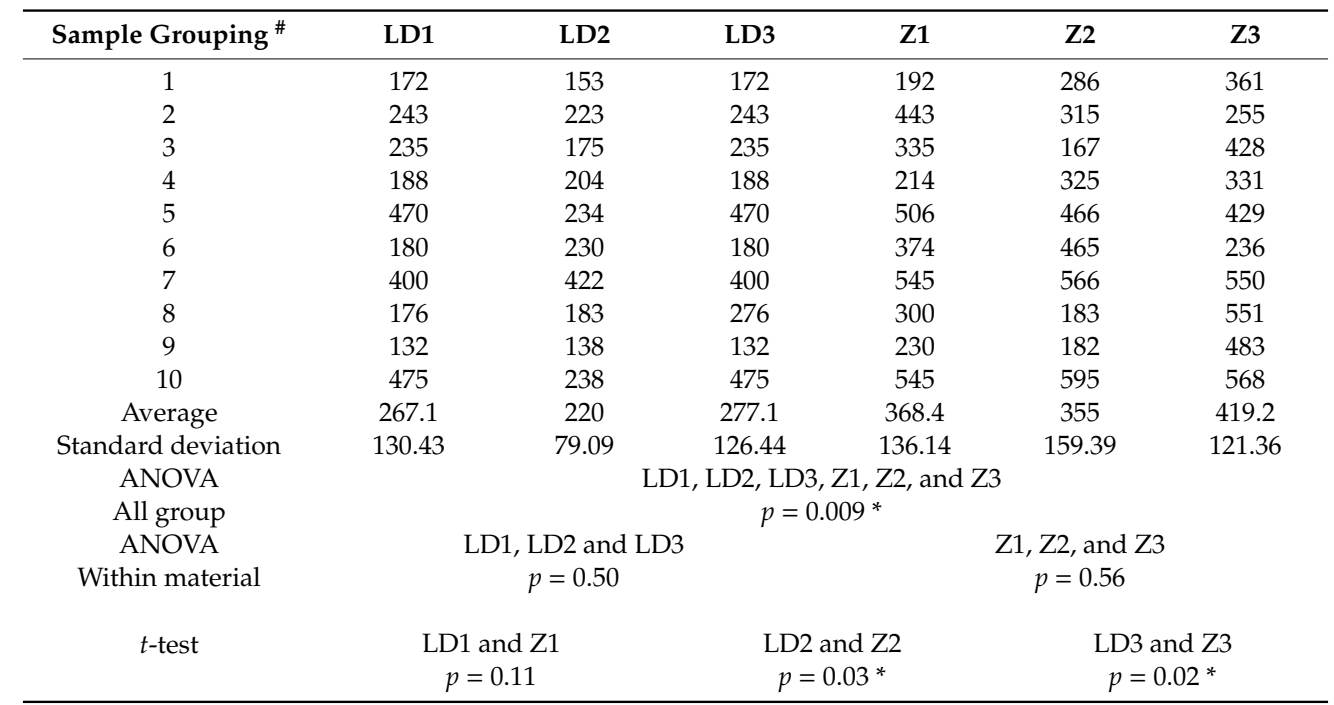

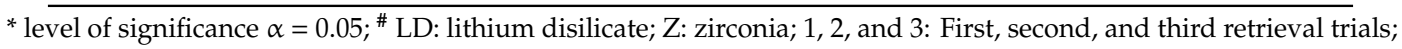
min: minute.

Table 2. Maximal temperatures $\left({ }^{\circ} \mathrm{C}\right)$ during Er:YAG laser irradiation.

\begin{tabular}{|c|c|c|c|c|c|c|}
\hline Sample Grouping \# & LD1 & LD2 & LD3 & $\mathrm{Z1}$ & $\mathrm{Z} 2$ & $\mathrm{Z3}$ \\
\hline 1 & 25 & 22.7 & 24.9 & 21 & 21.1 & 19.6 \\
\hline 2 & 23.7 & 23.7 & 26.8 & 21.4 & 24.2 & 22 \\
\hline 3 & 26.5 & 28.6 & 25 & 22.8 & 25 & 21.2 \\
\hline 4 & 27.3 & 23.6 & 23.2 & 20.5 & 27.8 & 21.7 \\
\hline 5 & 22.8 & 24.9 & 23.4 & 22.5 & 23.8 & 22 \\
\hline 6 & 24.1 & 25 & 24.1 & 23.2 & 22.7 & 22 \\
\hline 7 & 22.4 & 23.5 & 22.1 & 24.6 & 23.5 & 22.6 \\
\hline 8 & 22.1 & 28.2 & 25.3 & 24 & 23.9 & 21.5 \\
\hline 9 & 24.1 & 24.9 & 25 & 24.6 & 25 & 22.4 \\
\hline 10 & 21.5 & 23.5 & 21.9 & 24.2 & 23.3 & 24.9 \\
\hline Average & 23.95 & 24.86 & 24.17 & 22.88 & 24.03 & 21.99 \\
\hline Standard deviation & 1.89 & 2.01 & 1.53 & 1.51 & 1.74 & 1.32 \\
\hline ANOVA & \multicolumn{6}{|c|}{ LD1, LD2, LD3, Z1, Z2, and Z3 } \\
\hline All group & & & & & & \\
\hline ANOVA & \multirow{2}{*}{\multicolumn{3}{|c|}{ LD1, LD2 and LD3 }} & \multicolumn{3}{|c|}{$\mathrm{Z} 1, \mathrm{Z} 2$, and $\mathrm{Z} 3$} \\
\hline \multirow{2}{*}{$\begin{array}{c}\text { Within material } \\
t \text {-test }\end{array}$} & & & & \multicolumn{3}{|c|}{$p=0.02 *$} \\
\hline & \multicolumn{2}{|c|}{$\begin{array}{l}\mathrm{LD} 1 \text { and } \mathrm{Z} 1 \\
p=0.18\end{array}$} & \multicolumn{2}{|c|}{$\begin{array}{l}\mathrm{LD} 2 \text { and } \mathrm{Z} 2 \\
\quad p=0.34\end{array}$} & \multicolumn{2}{|c|}{$\begin{array}{l}\text { LD3 and Z3 } \\
p=0.003^{*}\end{array}$} \\
\hline
\end{tabular}

${ }^{*}$ level of significance $\alpha=0.05$; ${ }^{\#}$ LD: lithium disilicate; Z: zirconia; 1, 2, and 3: First, second, and third retrieval trials. 
The maximum temperatures for lithium disilicate were $23.95 .1 \pm 1.89^{\circ} \mathrm{C}, 24.86 \pm 2.01^{\circ} \mathrm{C}$, and $24.17 \pm 1.53^{\circ} \mathrm{C}$ for $\mathrm{LD} 1, \mathrm{LD} 2$, and LD3, respectively. The maximum temperatures for zirconia were $22.88 \pm 1.51^{\circ} \mathrm{C}, 24.03 \pm 1.74{ }^{\circ} \mathrm{C}$, and $21.99 \pm 1.32^{\circ} \mathrm{C}$ for $\mathrm{Z1}, \mathrm{Z} 2$, and $\mathrm{Z} 3$. There was a statistical difference overall (ANOVA, $p=0.006$ ) and within the zirconia group (ANOVA, $p=0.02$ ), while no statistical difference was noted within the lithium disilicate group (ANOVA, $p=0.52$ ). The maximum temperature was lower in the zirconia group compared to the lithium disilicate group; however, there was only statistical difference in the third retrieval experiment ( $t$-test, $p=0.18, p=0.34$, and $p=0.003$ for first, second, and third retrieval, respectively).

Changes in abutment surface area and cement gap as a result of additional tooth preparation prior to the third crown retrieval experiment are summarized in Table 3. The abutment surface area played an important role in the retrieval time in all cases $(r=0.67$ to $r=0.86)$. In terms of retrieval time, both materials demonstrated similar correlations. The larger surface area was positively correlated to the longer retrieval time. The cement gap played little to no role in the zirconia group $(r=0.2)$, while in the lithium disilicate group, the larger cement gap correlated with the faster retrieval time $(r=-0.43)$. In terms of temperature, the two materials behaved in the opposite direction with the weaker correlation compared to the retrieval time. Increasing the abutment surface reduced the temperature in the lithium disilicate group $(r=-0.45$ and $r=-0.48)$, but the opposite was seen in the zirconia group $(r=0.56$ and $r=0.69)$. The width of the cement gap had almost no role in the lithium disilicate group $(r=0.2)$, while the increased cement gap weakly increased the temperature in the zirconia group $(r=0.38)$.

Table 3. Effects of tooth modification on retrieval times and temperature.

\begin{tabular}{|c|c|c|c|c|c|c|}
\hline $\begin{array}{l}\text { Sample, Grouping, } \\
\text { and Surface } \\
\text { Area/Cement Gap }\end{array}$ & $\begin{array}{l}\text { Abutment } \\
\text { Surface LD1 } \\
\left(\mathrm{mm}^{3}\right)\end{array}$ & $\begin{array}{l}\text { Abutment } \\
\text { Surface LD3 } \\
\left(\mathrm{mm}^{3}\right)\end{array}$ & $\begin{array}{c}\text { Cement Gap } \\
\text { LD3 } \\
(\mu \mathrm{m})\end{array}$ & $\begin{array}{c}\text { Abutment } \\
\text { Surface Z1 } \\
\quad\left(\mathrm{mm}^{3}\right)\end{array}$ & $\begin{array}{c}\text { Abutment } \\
\text { Surface Z3 } \\
\left(\mathrm{mm}^{3}\right)\end{array}$ & $\begin{array}{c}\text { Cement Gap } \\
\text { Z3 } \\
(\mu \mathrm{m})\end{array}$ \\
\hline 1 & 127.67 & 119.75 & 394 & 133.81 & 125.69 & 504 \\
\hline 2 & 179.7 & 164.02 & 297 & 150.22 & 125.03 & 210 \\
\hline 3 & 152.55 & 122.87 & 360 & 138.57 & 124.54 & 359 \\
\hline 4 & 141 & 133.89 & 362 & 136.36 & 120.96 & 282 \\
\hline 5 & 226.38 & 209.80 & 277 & 182.76 & 160.93 & 251 \\
\hline 6 & 161.14 & 146.33 & 251 & 150.72 & 133.90 & 301 \\
\hline 7 & 218.57 & 209.31 & 335 & 193.97 & 173.70 & 291 \\
\hline 8 & 130.92 & 117.32 & 385 & 171.32 & 149.56 & 331 \\
\hline 9 & 131.21 & 121.22 & 407 & 136.45 & 122.48 & 302 \\
\hline 10 & 173.66 & 155.30 & 317 & 202.87 & 186.48 & 366 \\
\hline Average & 164.28 & 149.98 & 338.5 & 159.705 & 142.33 & 319.7 \\
\hline SD & 35.54 & 35.18 & 52.26 & 25.99 & 23.91 & 79.89 \\
\hline \multicolumn{7}{|l|}{ Pearson Correlation } \\
\hline $\begin{array}{l}\text { Each Factor and } \\
\text { Retrieval Time }\end{array}$ & 0.84 & 0.74 & -0.43 & 0.86 & 0.67 & 0.20 \\
\hline $\begin{array}{c}\text { Each Factor and } \\
\text { Retrieval Temperature }\end{array}$ & -0.45 & -0.48 & 0.20 & 0.56 & 0.69 & -0.38 \\
\hline
\end{tabular}

Visual examination of the crown and abutment tooth showed minor cement ablation and no visual cracks or damage to the material or tooth. The SEM analysis was made for one sample from each material group after each treatment (Figures 3 and 4 ). In the lithium disilicate and zirconia samples from all three experiments, some remaining cement particles were noted, however no noticeable surface damage, microcracks, or any other change was visible on the surface of teeth and crowns. Note that an additional lithium disilicate specimen was added and irradiated based on a published protocol proposed previously as the control [22]. 


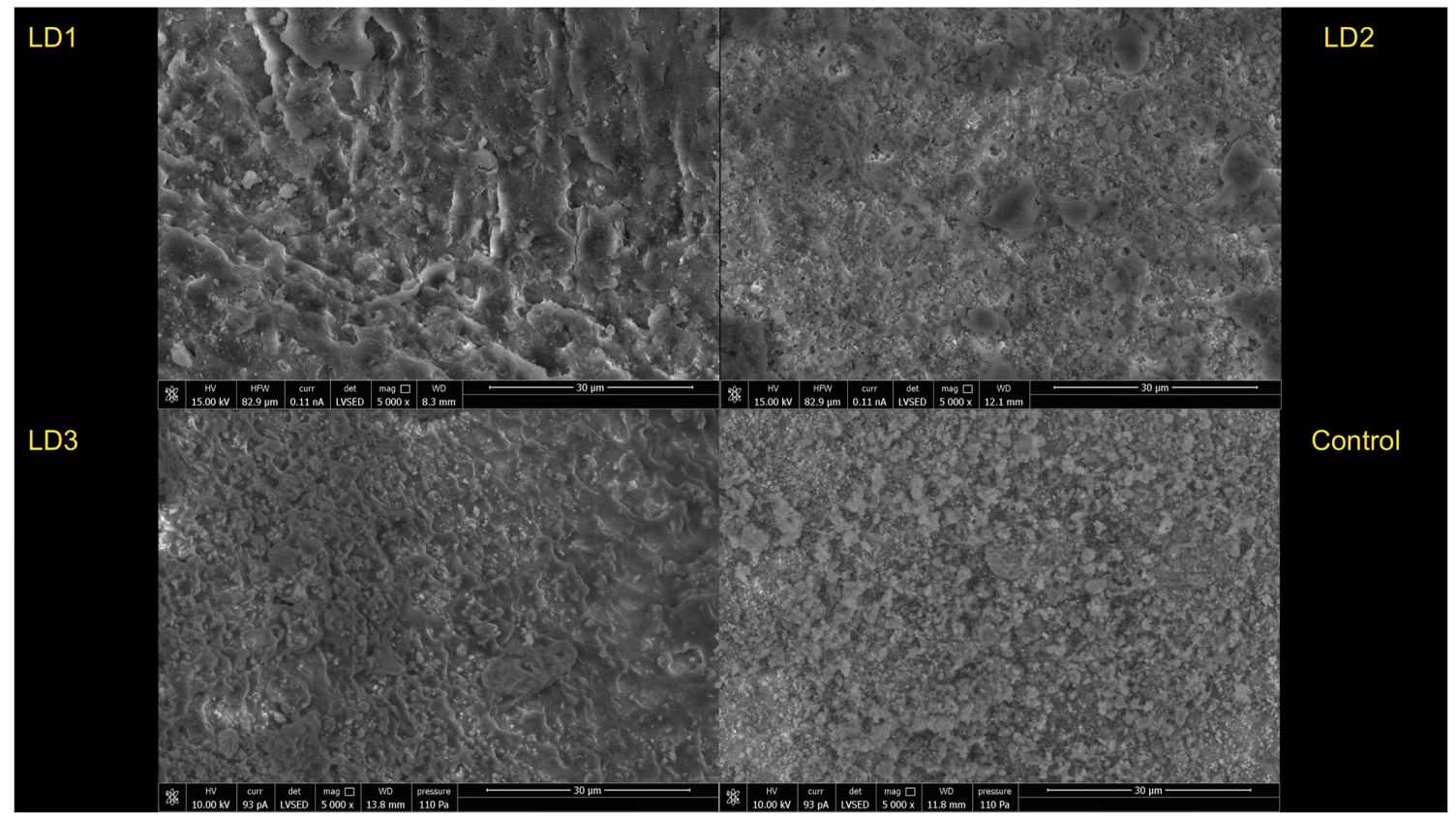

Figure 3. SEM analyses of lithium disilicate crowns. SEM of the cameo surface of lithium disilicate at the 1st (LD1), 2nd (LD2), 3rd (LD3), and a control of a higher laser setting based on a previous proposed protocol [22].

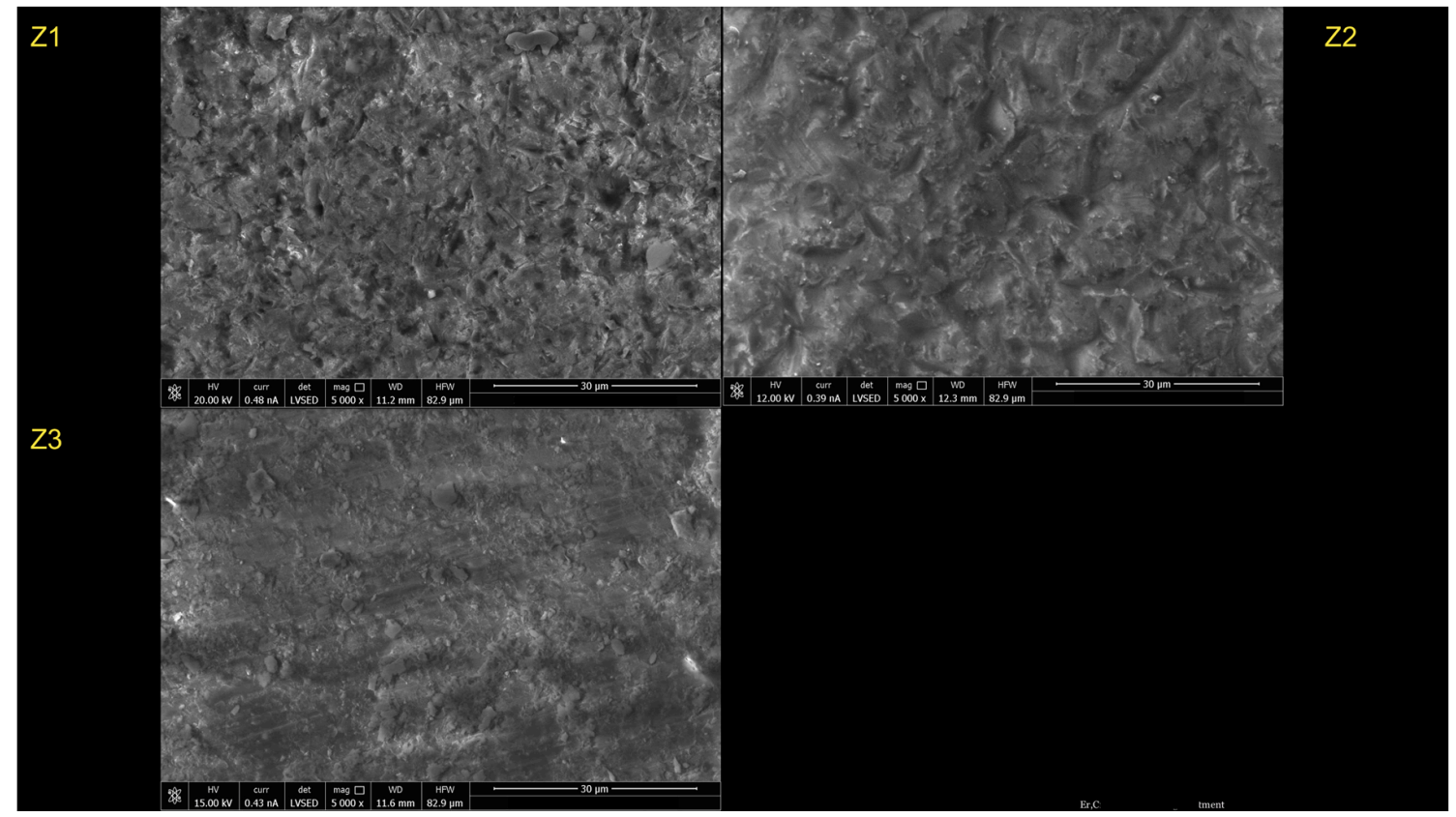

Figure 4. SEM analyses of zirconia crowns. SEM of the cameo surface of lithium disilicate at the 1st (Z1), 2nd (Z2), and 3rd (Z3).

\section{Discussion}

While some clinical reports and research on a non-invasive retrieval of all-ceramic crowns such as lithium disilicate and zirconia using Er:YAG laser exist in literature, there is little information on the different responses to laser-assisted crown retrieval from various ceramic materials. The results of this study highlighted the fact that crowns made from different ceramic materials behave differently when Er:YAG laser is used for their retrieval. As expected, lithium disilicate crowns are easier to retrieve than zirconia crowns. The time required to retrieve a lithium disilicate crown on average ranges from $3 \mathrm{~min}$ 
$40 \mathrm{~s}$ to $4 \mathrm{~min} 37 \mathrm{~s}$. The time required to retrieve a zirconia crown on average ranges from $5 \mathrm{~min} 55 \mathrm{~s}$ to $6 \mathrm{~min} 59 \mathrm{~s}$. Zirconia crowns therefore take 2-3 min longer to retrieve. These retrieval times align with findings from previous studies with the same laser settings [23,24]. The retrieval times for these natural teeth are slightly higher than retrieving a zirconia crown from zirconia implant abutment, which are approximately $5 \min 15 \mathrm{~s}$ to $5 \mathrm{~min} 20 \mathrm{~s}$ for the first time cementation and the repeated cementation [25]. This perhaps is a result of denser crystalized material of zirconia compared to less crystalized lithium disilicate. Furthermore, based on the study by Al-Juaila E et al. [26] lithium disilicate material shows higher translucency values than crystalline-based ceramics (zirconia), which can explain the results of this study. In theory, the laser can therefore penetrate through lithium disilicate better than zirconia [26]. This is reflected in longer retrieval time as well as $1{ }^{\circ} \mathrm{C}$ to $2{ }^{\circ} \mathrm{C}$ lower pulpal temperatures in the zirconia compared to the lithium disilicate group. It is interesting to note here that even the highest recorded temperature of $\sim 28^{\circ} \mathrm{C}$ in the pulpal chamber is still considered safe. The increased pulpal temperatures are also similar to increased temperature of zirconia implant abutment [24,25].

The increase in pulpal temperature over $5.5^{\circ} \mathrm{C}$ could cause irreversible damage to the pulp tissue [27]; however, temperature lower than $30^{\circ} \mathrm{C}$ is considered safe for the vitality of the dental pulp [27-30]. A rise in temperature to $50^{\circ} \mathrm{C}$ for $1 \mathrm{~min}$ on the root surfaces can cause irreversible damage to the periodontal ligament and bone that can lead to bone resorption and tooth ankylosis [31,32]. For both materials examined in this study, the temperature rises during crown retrieval using Er:YAG laser irradiation appear not harmful to the dental pulp or adjacent tissues. This study demonstrated that both lithium disilicate and zirconia crowns can be reused without compromised structural properties following re-retrieval with Er:YAG laser. These findings are consistent with previous observations on implant crowns [23,24]. The Er:YAG laser appears to have little or no effect on lithium disilicate material [33,34]. The laser settings used here appear to be too low to alter the structure of lithium disilicate or zirconia [23,24,35-37].

The goal of our study was also to achieve minimal retrieval time at the lowest possible settings to avoid potentially harmful temperature increases and irreversible damage to the tooth substance. Various authors have proposed a wide range of laser settings for debonding of restorations. While trying to determine optimal efficiency with safe parameters, the pilot experimented with a higher laser setting from a published protocol for retrieval of all-ceramic crowns $(508 \mathrm{~mJ}, 10 \mathrm{~Hz})$ [15,22], resulted in macroscopically noticeable significant damage to the abutment tooth. However, there was no damage seen by SEM in any of the specimens in the current study's protocol. The settings used in this study therefore represent safe and efficient laser settings for retrieval of lithium disilicate and zirconia crowns from natural teeth similar to the ones from implant abutments [23-25]. One difference was noted between the tooth and implant abutments; there seemed to be more cement left inside the zirconia crown retrieved from an implant abutment in the previous study [25] compared to those retrieved from the teeth. It is possible that the cement may adhere to natural tooth structure better than to the implant abutment made of zirconia.

Unique to this study, the third crown retrieval attempt was made after some adjustment of the tooth. This was intended to simulate a clinical situation when a dentist may need to adjust the abutment tooth to refresh the dentin to optimize bonding, to remove staining or dental caries, to remove residual cement, or to replace a part of missing tooth structure. The increased cement gaps proved to have little effect on the crown retrieval time or the temperature in the pulpal chamber. Larger abutment surface areas appear to have a positive relationship with the crown retention and therefore translate into the longer retrieval time. This implies that the most important factor for crown retention is the remaining bonding surface area of the abutment tooth structure.

There are some limitations to this study. First, the teeth selected for this study had good tooth structure and therefore allowed for an ideal preparation for a crown. In reality, teeth needing crowns have less optimal tooth structure rendering preparations less ideal, which may affect crown removal time. Second, attempts were made to simulate the oral cavity using a typodont mounted in a manikin head. In a live patient, cheeks and tongue can hinder the optimal access and direction of the laser 
irradiation. It may also be more challenging to get access and secure a crown tapping instrument at crown margins and at optimal angles. Finally, only one cement and one crown thickness (of 1-1.5 mm) were used in this study, different variations of cements and cementation protocols may alter the retrieval results. Future studies should focus on in vivo experiments to evaluate the retrieval time, different cementation protocols, different material thicknesses as well as clinical challenges and possibly patient's perceptions.

\section{Conclusions}

Lithium disilicate and zirconia crowns can be retrieved without any material or tooth damage using an Er:YAG laser. The retrieval time is longer for zirconia with lower temperature increases compared to lithium disilicate crowns. The pulpal temperature resulting from the laser irradiation appears to be within the physiological limit and likely has no adverse effect to the dental pulp or surrounding tissues. Er:YAG laser can be used safely and effectively for retrieval of common all-ceramic crowns.

Author Contributions: Conceptualization: J.G.D., S.B., and K.G.-L.; Data curation: S.B., K.G.-L., L.S., and D.K.; Formal analysis: S.B., K.G.-L., and J.M.; Investigation: J.G.D., S.B., K.G.-L., D.K., and L.S.; Methodology: J.G.D., S.B., K.G.-L., D.K., and L.S.; Project administration: S.B.; Visualization: S.B., L.S., and D.K.; Writing-original draft: S.B., J.G.D.; Writing-review \& editing: S.B., J.G.D., K.G.-L., D.K., and J.M. Authorship must be limited to those who have contributed substantially to the work reported. All authors have read and agreed to the published version of the manuscript.

Funding: This research received no external funding. The materials and staff were provided by the Virginia Commonwealth University Center of Digital Dentistry.

Acknowledgments: The authors thank the faculty and staff of the Virginia Commonwealth University Center of Digital Dentistry especially Marith Blacagon for the support in specimen preparation and material support.

Conflicts of Interest: The authors declare no conflict of interest.

\section{References}

1. Fasbinder, D.J.; Dennison, J.B.; Heys, D.; Neiva, G. A clinical evaluation of chairside lithium disilicate CAD/CAM crowns: A two-year report. J. Am. Dent. Assoc. 2010, 141 (Suppl. S2), 10-14. [CrossRef]

2. Aziz, A.; El-Mowafy, O.; Tenenbaum, H.C.; Lawrence, H.P.; Shokati, B. Clinical performance of chairside monolithic lithium disilicate glass-ceramic CAD-CAM crowns. J. Esthet. Restor. Dent. 2019, 31, $613-619$. [CrossRef]

3. Johansson, C.; Kmet, G.; Rivera, J.; Larsson, C.; Vult Von Steyern, P. Fracture strength of monolithic all-ceramic crowns made of high translucent yttrium oxide-stabilized zirconium dioxide compared to porcelain-veneered crowns and lithium disilicate crowns. Acta Odontol. Scand. 2014, 72, 145-153. [CrossRef]

4. Poticny, D.J.; Klim, J. CAD/CAM in-office technology: Innovations after 25 years for predictable, esthetic outcomes. J. Am. Dent. Assoc. 2010, 141 (Suppl. S2), 5-9. [CrossRef]

5. Wang, X.; Fan, D.; Swain, M.V.; Zhao, K. A systematic review of all-ceramic crowns: Clinical fracture rates in relation to restored tooth type. Int. J. Prosthodont. 2012, 25, 441-450. [PubMed]

6. Kassardjian, V.; Varma, S.; Andiappan, M.; Creugers, N.H.J.; Bartlett, D. A systematic review and meta analysis of the longevity of anterior and posterior all-ceramic crowns. J. Dent. 2016, 55, 1-6. [CrossRef] [PubMed]

7. Rodrigues, S.B.; Franken, P.; Celeste, R.K.; Leitune, V.C.B.; Collares, F.M. CAD/CAM or conventional ceramic materials restorations longevity: A systematic review and meta-analysis. J. Prosthodont. Res. 2019, 63, 389-395. [CrossRef] [PubMed]

8. Guarda, G.B.; Correr, A.B.; Gonçalves, L.S.; Costa, A.R.; Borges, G.A.; Sinhoreti, M.A.C.; Correr-Sobrinho, L. Effects of surface treatments, thermocycling, and cyclic loading on the bond strength of a resin cement bonded to a lithium disilicate glass ceramic. Oper. Dent. 2013, 38, 208-217. [CrossRef] [PubMed]

9. Brum, R.; Mazur, R.; Almeida, J.; Borges, G.; Caldas, D. The influence of surface standardization of lithium disilicate glass ceramic on bond strength to a dual resin cement. Oper. Dent. 2011, 36, 478-485. [CrossRef] [PubMed]

10. Atay, A.; Sagirkaya, E. Effects of different surface treatments on the bond strength of CAD/CAM resin nano ceramic or ceromer to resin cement. Cumhur. Dent. J. 2019, 22, 226-234. [CrossRef] 
11. Al-Amleh, B.; Lyons, K.; Swain, M. Clinical trials in zirconia: A systematic review. J. Oral. Rehabil. 2010, 37, 641-652. [CrossRef] [PubMed]

12. Øilo, M.; Kvam, K.; Gjerdet, N.R. Load at fracture of monolithic and bilayered zirconia crowns with and without a cervical zirconia collar. J. Prosthet. Dent. 2016, 115, 630-636. [CrossRef] [PubMed]

13. Moaleem, M.M.A.; Al Moaleem, M.M. Systems and techniques for removal of failed fixed partial dentures: A Review. Am. J. Health Res. 2016, 4, 109. [CrossRef]

14. Bajunaid, S.O. Review of techniques for the intact removal of a permanently cemented restoration. Gen Dent. 2017, 65, 48-53. [PubMed]

15. Rechmann, P.; Buu, N.C.H.; Rechmann, B.M.T.; Finzen, F.C. Laser all-ceramic crown removal-a laboratory proof-of-principle study-Phase 2 crown debonding time. Lasers Surg. Med. 2014, 46, 636-643. [CrossRef] [PubMed]

16. Rechmann, P.; Buu, N.C.H.; Rechmann, B.M.T.; Finzen, F.C. Laser all-ceramic crown removal and pulpal temperature-a laboratory proof-of-principle study. Lasers Med. Sci. 2015, 30, 2087-2093. [CrossRef] [PubMed]

17. Lizarelli, R.F.Z.; Moriyama, L.T.; Bagnato, V.S. Ablation of composite resins using Er:YAG laser-comparison with enamel and dentin. Lasers Surg. Med. 2003, 33, 132-139. [CrossRef]

18. Morford, C.K.; Buu, N.C.H.; Rechmann, B.M.T.; Finzen, F.C.; Sharma, A.B.; Rechmann, P. Er:YAG laser debonding of porcelain veneers. Lasers Surg Med. 2011, 43, 965-974. [CrossRef]

19. Buu, N.; Morford, C.; Finzen, F.; Sharma, A.; Rechmann, P. Er:YAG laser debonding of porcelain veneers. In Proceedings of the SPIE BiOS, San Francisco, CA, USA, 23 January 2010. [CrossRef]

20. Dostalova, T.; Jelinkova, H.; Remes, M.; Šulc, J.; Němec, M. The use of the Er:YAG laser for bracket debonding and its effect on enamel damage. Photomed. Laser Surg. 2016, 34, 394-399. [CrossRef]

21. Grzech-Leśniak, K.; Matys, J.; Żmuda-Stawowiak, D.; Mroczka, K.; Dominiak, M.; Junior, A.B.; Gruber, R.; Romanos, G.E.; Sculean, A. Er:YAG laser for metal and ceramic bracket debonding: An in vitro study on intrapulpal temperature, SEM, and EDS analysis. Photomed. Laser Surg. 2018, 36, 595-600.

22. Kellesarian, S.V.; Ros Malignaggi, V.; Aldosary, K.M.; Javed, F. Laser-assisted removal of all ceramic fixed dental prostheses: A comprehensive review. J. Esthet. Restor. Dent. 2018, 30, 216-222. [CrossRef] [PubMed]

23. Grzech-Leśniak, K.; Bencharit, S.; Dalal, N.; Mroczka, K.; Deeb, J.G. In vitro examination of the use of Er:YAG laser to retrieve lithium disilicate crowns from titanium implant abutments. J. Prosthodont. 2019, 28, 672-676. [CrossRef] [PubMed]

24. Deeb, J.G.; Bencharit, S.; Dalal, N.; Abdulmajeed, A.; Grzech-Leśniak, K. Using Er:YAG laser to remove lithium disilicate crowns from zirconia implant abutments: An in vitro study. PLoS ONE 2019, 14, e0223924.

25. Elkharashi, A.; Grzech-Leśniak, K.; Deeb, J.G.; Abdulmajeed, A.A.; Bencharit, S. Exploring the use of pulsed erbium lasers to retrieve a zirconia crown from a zirconia implant abutment. PLOS ONE 2020, 15, e0233536.

26. Al-Juaila, E.; Osman, E.; Segaan, L.; Shrebaty, M.; Farghaly, E.A. Comparison of translucency for different thicknesses of recent types of esthetic zirconia ceramics versus conventional ceramics ... (in vitro study). Future Dent. J. 2018, 42, 297-301. [CrossRef]

27. Zach, L.; Cohen, G. Pulp response to externally applied heat. Oral Surg. Oral Med. Oral Pathol. 1965, 19, 515-530. [CrossRef]

28. d'Ornellas Pereira, J.C., Jr.; Weissheimer, A.; de Menezes, L.M.; de Lima, E.M.S.; Mezomo, M. Change in the pulp chamber temperature with different stripping techniques. Prog. Orthod. 2014, 15, 55. [CrossRef]

29. Ramoglu, S.I.; Karamehmetoglu, H.; Sari, T.; Usumez, S. Temperature rise caused in the pulp chamber under simulated intrapulpal microcirculation with different light-curing modes. Angle Orthod. 2015, 85, 381-385. [CrossRef]

30. Laforgia, P.D.; Milano, V.; Morea, C.; Desiate, A. Temperature change in the pulp chamber during complete crown preparation. J. Prosthet. Dent. 1991, 65, 56-61. [CrossRef]

31. Eriksson, A.R.; Albrektsson, T. Temperature threshold levels for heat-induced bone tissue injury: A vital-microscopic study in the rabbit. J. Prosthet. Dent. 1983, 50, 101-107. [CrossRef]

32. Eriksson, R.A.; Albrektsson, T.; Magnusson, B. Assessment of bone viability after heat trauma. A histological, histochemical and vital microscopic study in the rabbit. Scand. J. Plast. Reconstr. Surg. 1984, 18, 261-268. [CrossRef] [PubMed]

33. Viskic, J.; Jokic, D.; Jakovljevic, S.; Bergman, L.; Ortolan, S.M.; Mestrovic, S.; Mehulic, K. Scanning electron microscope comparative surface evaluation of glazed-lithium disilicate ceramics under different irradiation settings of Nd:YAG and Er:YAG lasers. Angle Orthod. 2018, 88, 75-81. [CrossRef] [PubMed] 
34. Yamada, M.K.; Uo, M.; Ohkawa, S.; Akasaka, T.; Watari, F. Three-dimensional topographic scanning electron microscope and Raman spectroscopic analyses of the irradiation effect on teeth by Nd:YAG, Er: YAG, and CO2 lasers. J. Biomed. Mater. Res. 2004, 71, 7-15. [CrossRef] [PubMed]

35. Stübinger, S.; Homann, F.; Etter, C.; Miskiewicz, M.; Wieland, M.; Sader, R. Effect of Er:YAG, CO(2) and diode laser irradiation on surface properties of zirconia endosseous dental implants. Lasers Surg. Med. 2008, 40, 223-228. [CrossRef] [PubMed]

36. Turp, V.; Akgungor, G.; Sen, D.; Tuncelli, B. Evaluation of surface topography of zirconia ceramic after Er:YAG laser etching. Photomed. Laser Surg. 2014, 32, 533-539. [CrossRef] [PubMed]

37. Gökçe, B.; Ozpinar, B.; Dündar, M.; Cömlekoglu, E.; Sen, B.H.; Güngör, M.A. Bond strengths of all-ceramics: Acid vs laser etching. Oper. Dent. 2007, 32, 173-178. [CrossRef]

(C) 2020 by the authors. Licensee MDPI, Basel, Switzerland. This article is an open access article distributed under the terms and conditions of the Creative Commons Attribution (CC BY) license (http://creativecommons.org/licenses/by/4.0/). 